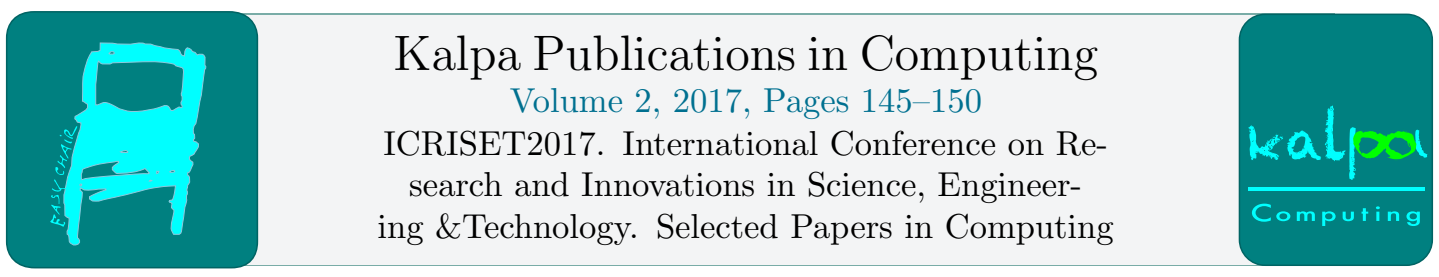

\title{
An open source real time IoT based environmental sensor monitoring system
}

\author{
Ronak Vithlani ${ }^{2}$, Siddharth Fultariya ${ }^{2}$, Mahesh Jivani ${ }^{1}$, Haresh Pandya ${ }^{1}$ \\ ${ }^{1}$ Professor, Department of Electronics, Saurashtra University, Rajkot 360005, Gujarat, India \\ ${ }^{2}$ Research Scholar, Department of Electronics, Saurashtra University, Rajkot 360005, Gujarat. \\ rk.vithlani@live.com, fultariyas@gmail.com, \\ mnjivani@sauuni.ernet.in, hnpandya@yahoo.com
}

\begin{abstract}
In this paper, we have described an operative prototype for Internet of Things (IoT) used for consistent monitoring various environmental sensors by means of low cost open source embedded system. The explanation about the unified network construction and the interconnecting devices for the consistent measurement of environmental parameters by various sensors and broadcast of data through internet is being presented. The framework of the monitoring system is based on a combination of embedded sensing units, information structure for data collection, and intellectual and context responsiveness. The projected system does not involve a devoted server computer with respect to analogous systems and offers a light weight communication protocol to monitor environment data using sensors. Outcomes are inspiring as the consistency of sensing information broadcast through the projected unified network construction is very much reliable. The prototype was experienced to create real-time graphical information rather than a test bed set-up.
\end{abstract}

\section{Introduction}

Automated environmental sensor monitoring system is the key of contemporary farming. Farming facility job is rising now a days and automation adds on the worth to agricultural sustainable progress. Traditional agricultural progress cannot be satisfied the current necessities of agricultural sustainable progress. For shifting the traditional mode of the agricultural system to projected system, the application of internet of things (IoT) in greenhouse automation is the key arena in present days. Application and implementation of IoT can decrease the main hassle of men made monitoring system and manpower. IoT based smart remote monitoring of the environmental data such as humidity, temperature, air pollution, water and soil wetness in plants are obtained in real time. 
IoT is a network of Internet empowered things in a universe where physical objects are flawlessly combined into the information network and where the physical objects can become lively contributors in corporate developments (Carretero, 2014). The Internet of Things (Atzori L, 2010) brings together two key perceptions: Internet-connected devices ubiquitously in any time and any place and pervasive computing, where "the utmost profound technologies are those that disappear" (Weiser, 1999) in such a way that these devices made themselves indistinguishable from explicit technology that the humans use in their lives. The presented system holds sensor system that contains several applications based sensors, embedded network, information platform and satellite network that forms a huge network (Jagdale, 2016). This can please the necessity of cutting-edge automated farming production through the support of various environmental sensor nodes which gather environmental parameters such as temperature, humidity, carbon dioxide, wetness level, oxygen level. Wi-Fi enabled open sourced Embedded system connected with internet facility which broadcasts said parameters on open source IoT platform www.thinger.io (Thinger.io, An open source IoT Platform, n.d.) which presents real time data graphically through website as well as mobile applications forms satellite network to avail data from anywhere.

Jalpa Shah and Bisvajit Mishra presented a customized design of an Internet of Things (IoT) enabled environment monitoring system to monitor temperature, humidity and CO2 using LabView, where data is sent from the transmitter node to the receiver node. The data received at the receiver node is monitored and recorded in an excel sheet in a personal computer (PC) through a Graphical User Interface (GUI), made in LabVIEW (Shah, 2016). Azzatdin A. A. uses GSM short message technology to understand real time controlling of greenhouse (Aziz, 2009). Ting Chau Lu et al presented a self-sustainable landslide surveillance system that detects hazardous water content level in soils and provides real-time landslide warnings to residents, without requiring wired electricity transmission using environmental sensor monitoring system (Lu, 2016). Imaran B. Z. presented wireless monitoring system using nRF24L01 and database using Microsoft visual studio (Jafar, 2014).

\section{Experimental Setup}

The presented system provides environmental sensor monitoring in real time and it meets the requirement of reliability and sustainability. In present system, a wireless sensor network with

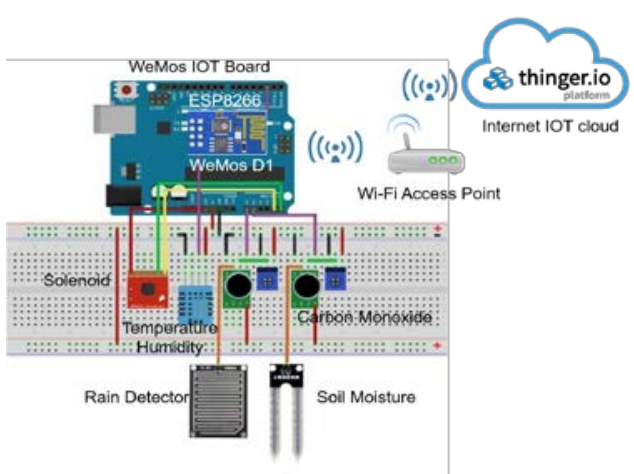

Figure 1: Experimental Setup embedded controlling system was designed and implemented for the environmental wireless sensor network monitoring and Management. It can sense significant environmental Maintaining the Integrity of the Specifications parameters like Temperature, Humidity, Soil moisture, water and soil wetness for plants in real time with the help of sensors and wireless embedded system. System broadcast sensors' data wirelessly with the help of an WeMos D1 R2 board (WeMos D1 R2 Embbedded IoT Board based on ESP8266EX, n.d.) Compatible Wi-Fi board based on ESP8266EX) through internet connection to free open source IoT web platform www.thinger.io for further analysis. The environmental parameters like Temperature, Humidity and soil moisture are controlled by the entire system. The sensing network runs by WeMos D1 R2 board which is very easily programmed through Arduino IDE (Using AVR Studio for Arduino development, n.d.). 
For communication purpose, ESP8266EX Wi-Fi board connected to Wi-Fi access point for internet accessibility and to cloud for further analysis. The outputs of the sensors are given as input to the ESP8266EX micro controller to control and update the dashboard of thinger.io which configures parameter of environmental sensor and presents on display as textually as well as graphically.

\subsection{WeMos D1 R2 IoT Board}

WeMos-D1R2 IoT board is an ESP8266EX based Wi-Fi aided microcontroller piece on an ArduinoUNO footprint. It means the board appearances and functions (in utmost occasions) like an UNO. Seemingly, some shields, sensors and output devices that are manufactured for the Arduino platform will work on the WeMos-D1R2 board with the additional benefit of integral Wi-Fi. ESP8266EX controller works on $3.3 \mathrm{~V}$ and has the clock speed of $80 \mathrm{MHz}$ with $4 \mathrm{MB}$ of program storage with $11 \mathrm{I} / \mathrm{O}$ pins, excluding D0; all pins have interrupt/PWM/I2C/one-wire supported, 1 analog input (3.2V max input), Micro USB connection, Compatible with Arduino, built in Wi-Fi, on board antenna and most beautiful part is it available at low cost.

\subsection{Sensors}

Environmental Monitoring System uses various types of sensors which are described briefly here:

\section{Temperature and Humidity DHT11 Sensor}

The DHT11 Temperature \& Humidity Sensor provides a temperature \& humidity sensor compound with a calibrated digital signal output. It uses the high-class digital-signal-acquisition method and temperature \& humidity sensing technology, it guarantees great consistency and outstanding long-term steadiness. DHT11 sensor comprises a resistive-type humidity measurement component and an NTC temperature measurement component.

\section{Carbon Monoxide MQ7 Sensor}

Carbon Monoxide (CO) MQ7 gas sensor senses the concentrations of $\mathrm{CO}$ in the air and outputs its data as an analog voltage. MQ7 sensor can measure concentrations of 10 to $10,000 \mathrm{ppm}$ and can function at temperatures from -10 to $50^{\circ} \mathrm{C}$ and consumes fewer than $150 \mathrm{~mA}$ at $5 \mathrm{~V}$. The Subtle material of MQ-7 gas sensor is $\mathrm{SnO} 2$, which have lower conductivity in clean air. It makes detection by method of cycle high and low temperature, and detect CO when low temperature (heated by $1.4 \mathrm{~V}$ ). The sensor's conductivity is higher along with the gas concentration rising. For high temperature (heated by 5.0V), it cleans the other gases adsorbed under low temperature.

\section{Sprinkle rain Sensor}

The Sprinkle rain sensor senses water which completes the circuits on its sensor boards' printed leads. The sensor board works as a variable resistor that will change from $100 \mathrm{k}$ ohms when wet to $2 \mathrm{M}$ ohms when dry. In short, the wetter the board the more current that will be conducted. A sprinkler rain sensor functions via a gauge mounted on an exterior arm, that is fixed on a fence near flower plant, lawn or garden, connected to the sprinkler system. Plate inside the gauge detects water, these send a signal to the sprinkler system controller, intruding the electronic signal that turns off the sprinklers. The signal is OFF until the plate dry again. The sprinkler controller then receives the start signal, and resumes its spraying schedule.

\section{Solenoid Valve}

A solenoid valve, generally identified as an electrically-operated valve is an automatic valve which serves the purpose of eliminating the requirement for a person to operate a valve physically. 
An electromagnetic solenoid coil to alter the state of a valve from closed to open, or reverse operated by Solenoids. If the solenoid valve is 'normally closed', when the coil is energized, the valve gets lifted open by the electromagnetic force produced by the coil. Here, in this work, when plate of sprinkler rain sensor gets dry it sends the signal to microcontroller board to energized the coil and the valve gets lifted open and sprinkler can spray or pour the water to plants automatically.

\subsection{Software IDE}

The heart of WeMos D1 R2 board is ESP8266EX Wi-Fi enabled microcontroller which can easily be programmed through an open source Arduino IDE. The open-source Arduino environment makes it easy to write code and upload it to the I/O board. It runs on Windows, Mac OS X, and Linux. The environment is written in Java and it is based on Processing, avr-gcc, and other open source software. The Arduino development environment contains a text editor for writing code, message area, text console, and toolbar with buttons for common functions, and a series of menus. It connects to the Arduino hardware to upload programs and communicate with them. Arduino programs are written in $\mathrm{C}$ or $\mathrm{C}++$. Arduino IDE with features such as syntax highlighting, brace matching, and automatic indentation, and it is also capable of compiling and uploading programs to the Board with a single click (Arduino Development Environment, n.d.). Software written using Arduino are called sketches. These sketches are written in the text editor. Sketches are saved with the file extension '. ino.' It has features for cutting/pasting and for searching/replacing text. The message area gives feedback while saving and exporting and also displays errors. The console displays text output by the Arduino environment including complete error messages and other information. The bottom right-hand corner of the window displays the current board and serial port. The toolbar buttons allow you to verify and upload programs, create, open, and save sketches, and open the serial monitor (Arduino IDE, n.d.). As the Arduino platform uses Atmel microcontrollers, Atmel's development environment AVR Studio or the newer Atmel Studio, may also be used to develop software for the Arduino (Using Atmel Studio for Arduino development, n.d.).

\section{SOFTWARE DETAILS}

The program for embedded board WeMos D1 R2 board is prepared and uploaded using Arduino IDE. As shown in flow chart (Fig.2), the inbuilt ESP8266EX wi-fi module is initialized as wi-fi access point using ESP8266WiFi.h library which initiates the SSID login and password using serial access provided by SPI.H library.

Thinger.io library initialize the objects of rest API for binding device with parameters like 'user_name', 'DEVICE_ID' and 'Device_Credential' which provides connection between ESP8266 board and processing engine of Thinger.io IoT platform. Environmental sensors connected with EXP8266 board provides various resource parameters for processing data to Thinger.io and each resource are controlled and displayed individually with the help of dashboard at desired sampling interval.

\section{IOT PLATFORM: THINGER.IO}

Thinger.io is the open source platform for the internet of the things application and API to store as well as retrieve the data from things using 'protoson' formatted HTTP protocol over the internet. Thinger.io enables the establishment of sensor logging application and social network of things with current status updates [5]. The IoT offers access to a wide range of embedded devices and web services. It can allow 
to primary collect, visualize, analyze, and turn on data from sensors or actuators with various compatible embedded boards.

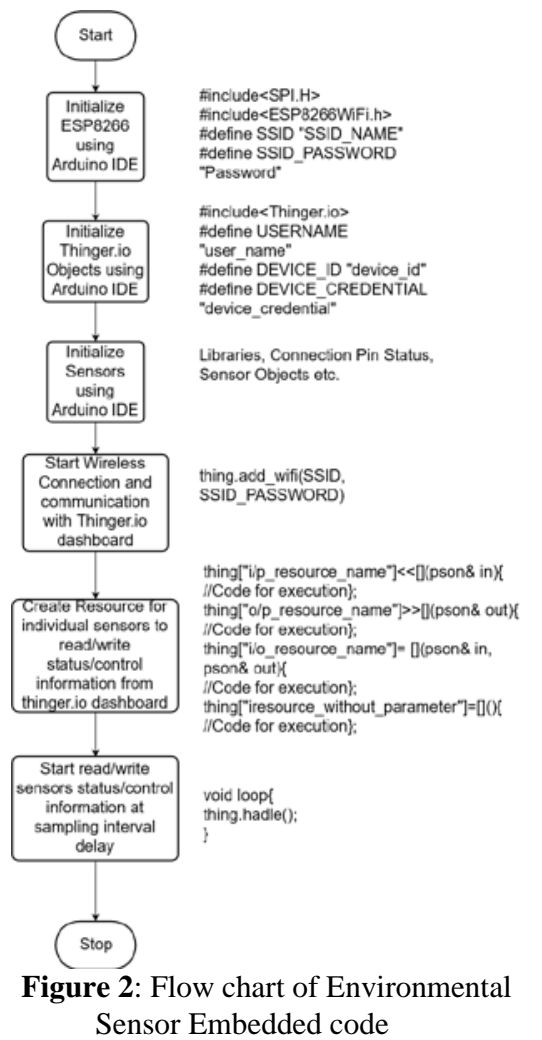

The utmost significant part of Thinger.io service is the dashboard which contains various widget of devices, data fields, location fields, and a status field. After creating a Thinger.io dashboard one can add the device, write data to the dashboard, process and view the data. Information can be presented through numerically or graphically and it also provides alerts through its rest APIs. The Thinger.io workflow as follows:

- Create the dashboard for particular device and acquired the data

- Analyze the data by selecting various permission and parameters

- Present the data by selecting various alert of time stamp as well as graphical facility

The Thinger.io includes the complete rest API for dealing out HTTP requests, stores alphanumeric data, numeric and processing, location tracking, and status updates (Thinger.io, An open source IoT Platform, n.d.). For creating a new dashboard one needs to sign in and required API key for logging the data. The sensor parameters of different field like temperature, Humidity, Carbon Monoxide Level and Solenoid ON/OFF status are displayed on Thinger.io website in real time. The Thiger.io also provides and mobile application. This application allows controlling IoT

devices connected to the https://thinger.io platform. It requires an account or scan a device token in order to use it. At this moment, mobile app is in preliminary stage, which provides only numeric information.

\section{IMPLEMENTATION}

Power up the Circuit and check status of blinking Inbuilt LED of WeMos D1 R2 board for the availability of Wi-Fi network. If everything is done correctly, you will be able to see stable LED and Wi-Fi Network which is connected to internet enabled access point.

Follow the steps listed below for monitoring and controlling various parameter of environment sensors.

- Open the www.thinger.io with user credential on your access device

- Open Device Tab and check it is connected or not

- For monitoring status of various sensors, open dashboard tab and check the data in form of various forms of graphs and status. 


\section{CONCLUSION}

The present work is accountable for information collection, transmission and the environmental information intelligent processing by means of Internet of things. One can check the actual environmental filed parameter on Thinger.io website and access remotely from anywhere on the world using internet facility with secured token provided by Things.io Platform for website and mobile devices. Use of the Internet of things in automated environmental data monitoring system improves the efficiency of the greenhouses automation, crop production with less manpower and protection of greenhouse environment. The Environmental Sensor monitoring system is also tested using Android mobile phone application provided by Thinger.io which are quite satisfactory and responses received from the community in general are encouraging. The Environmental Sensor Monitoring system furnishes a good paradigm for any Automation System based on Internet of Things (IoT).

\section{References}

Arduino Development Environment. (n.d.). Retrieved from http:// arduino.cc / en / guide / Environment. Arduino IDE. (n.d.). Retrieved from http://arduino.cc/en/main/software.

Atzori L, I. A. (2010). The Internet of Things: a survey. Computer networks, 54(15), 2787-2805.

Aziz, I. A. (2009). Remote monitoring in agricultural greenhouse using wireless sensor and short message service (SMS). International Journal of Engineering \& Technology IJET, 9(9), 1-12.

Carlisle, D. (2010, April). graphicx: Enhanced support for graphics. Retrieved from http://www.ctan.org/tex-archive/ help/Catalogue/entries/graphicx.html

Carretero, J. a. (2014). The Internet of Things: connecting the world. Personal and Ubiquitous Computing, 18(2), 445-447.

Jafar, I. B. (2014). Wireless monitoring system and controlling software for Smart Greenhouse Management. International Conference on Informatics, Electronics \& Vision (ICIEV) (pp. 15). IEEE.

Jagdale, T. a. (2016, January). Greenhouse Wireless Network Monitoring and Management Using IoT. Iternational journal of Innovative Research in Electrical, Electronics, Instrumentation and Control Engineering, 4(1). doi:doi: 10.17148 /IJIREEICE.2016.4105

$\mathrm{Lu}, \mathrm{T}$. H. (2016). Invited-Wireless sensor nodes for environmental monitoring in internet of things. In Proceedings of the 53rd Annual Design Automation Conference (ACM), (p. 3).

Shah, J. a. (2016). IoT enabled environmental monitoring system for smart cities. International Conference on Internet of Things and Applications (IOTA) (pp. 383-388). IEEE.

Thinger.io, An open source IoT Platform. (n.d.). Retrieved from www.thinger.io.

Using Atmel Studio for Arduino development. (n.d.). Retrieved from www.megunolink.com.

Using AVR Studio for Arduino development. (n.d.). Retrieved from www.engblaze.com.

Weiser, M. (1999). The computer for the 21st century. Mobile Computing and Communications Review, 3(3), 3-11.

WeMos D1 R2 Embbedded IoT Board based on ESP8266EX. (n.d.). Retrieved from www.wemos.cc . 